\title{
2-Photon Laser Scanning Microscopy on Native Human Cartilage
}

\author{
Jörg Martini, Katja Tönsing, Michael Dickob*, Dario Anselmetti \\ Bielefeld University, Physics Faculty, Experimental BioPhysics \& Applied NanoScience, \\ Universitätsstr. 25, 33615 Bielefeld, Germany \\ * Orthopedic Surgery, Bahnhofstrasse 30, 33602 Bielefeld, Germany
}

\begin{abstract}
Native hyaline cartilage from a human knee joint was directly investigated with laser scanning microscopy via 2-photon autofluorescence excitation with no additional staining or labelling protocols in a nondestructive and sterile manner. Using a femtosecond, near-infrared (NIR) Ti:Sa laser for 2-photon excitation and a dedicated NIR long distance objective, autofluorescence imaging and measurements of the extracellular matrix (ECM) tissue with incorporated chondrocytes were possible with a penetration depth of up to $460 \mu \mathrm{m}$ inside the sample. Via spectral autofluorescence separation these experiments allowed the discrimination of chondrocytes from the ECM and therefore an estimate of chondrocytic cell density within the cartilage tissue to approximately $0.2-2 \cdot 10^{7} / \mathrm{cm}^{3}$. Furthermore, a comparison of the relative autofluorescence signals between nonarthritic and arthritic cartilage tissue exhibited distinct differences in tissue morphology. As these morphological findings are in keeping with the macroscopic diagnosis, our measurement has the potential of being used in future diagnostic applications.
\end{abstract}

Keywords: three-dimensional microscopy; multiphoton processes, biomedical imaging, human cartilage

\section{INTRODUCTION \& MOTIVATION}

Hyaline cartilage consists of chondrocytes that are embedded in an extracellular matrix tissue. The ECM, that was originally formed and is maintained by chondrocytes, contains mostly collagen II, proteogylcans like chondroitin sulfate and keratan sulfate, glucosamine, and water (Figure 1). Earlier experiments have exhibited that the chondrocytes are located in tissue cavities (lacunae) and often group during their proliferation [1,2]. The three dimensional collocation of the lacunae is therefore dependent on their relative position in the cartilage, i.e. distance to the bone. Degeneration of
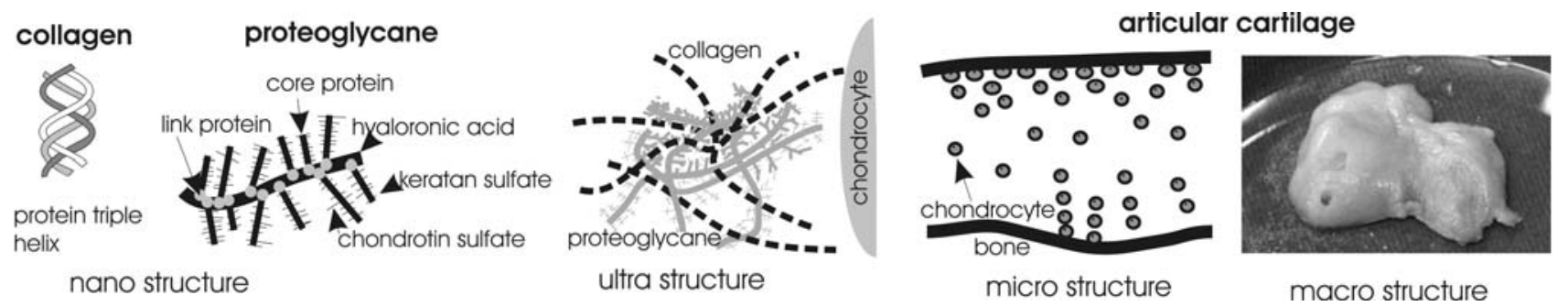

Figure 1: (from left to right) Nano-, ultra- und microstructure of human knee joint cartilage with severe arthritic lesion on the right. 
cartilage (arthritis) is due to a deterioration of ECM, water content and chondrocytes and its loss of intrinsic elasticity. Due to the intrinsically dense structure of the ECM, cartilage is a tissue that strongly scatters but does not absorb much light. Hence articular cartilage has a white optical appearance and is called hyalin. Consequently, penetration depth of visible light is vastly reduced, making a characterization with conventional brightfield microscopy extremely difficult, as they require staining and/or microtome cuts. A remedy is using NIR light that is less affected by light scattering and is able to deeply penetrate such biological tissue. Combining this advantage with the benefit of NIR two-photon excitation [3] (Figure 2) allows a proper 3-dimensional microscopic characterization of these tissues. As the 2-photon excitation wavelength of the laser can be tuned, inducing autofluorescence of the sample (i.e. cartilage), staining protocols become obsolete. However, two-photon excitation depends on extremely high photon densities that can only be realized by an adequate spatial and temporal focussing of light, such as in a diffraction-limited focus of a femtosecond laser beam.

\section{(a)}

\section{(b)}

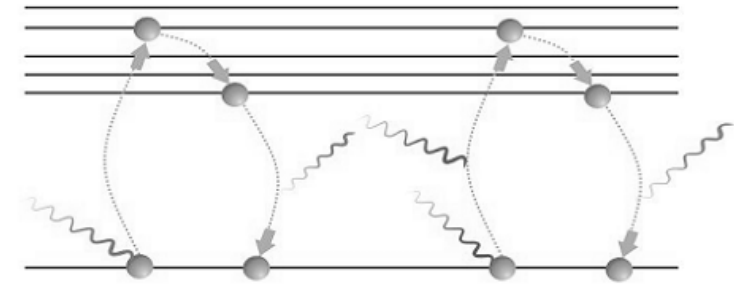

Figure 2: (a) Conventional one-photon excitation (b) two-photon excitation

Therefore, in this paper, we investigate the application of two-photon laser scanning microscopy (2PLSM) for the characterization of human cartilage tissue with respect to the following questions: 1) is an adequate and stain-free imaging of the cartilage tissue possible with 2PLSM, 2) can chondrocytic cells be identified and discriminated from ECM spectrally, and 3) is there a detectable difference that can be used in the future for optical diagnostic evaluation of healthy and arthritic cartilage tissue.

\section{EXPERIMENTAL SETUP}

\subsection{Two-Photon Laser Scanning Microscope}

Our 2-photon-laser-scanning-microscope (2PLSM) consists of a mode locked Tsunami Ti:Sa laser, pumped by a Millenia X solid-state laser (both Spectra-Physics) that generates $100 \mathrm{fs}$ laser pulses between 760nm and 960nm (see Figure 3, \#1), a TriM Scope scanning unit (La Vision Biotec, see Figure 3, \# 2, 3, 4) and an inverted microscope (IX 71, Olympus, see Figure 3, \#5). Detection of the fluorescence signal is realized by a back illuminated EMCCD camera (IXON BV887ECS-UVB, Andor Technology; see Figure 3, \#b) or a photomultiplier (H7422-40, Hamamatsu; see Figure 3, $\# \mathrm{c}$ ) in a nondescanned manner. Therefore, the NIR laserbeams, used for excitation, are being directed with a dichroic mirror (2P-Beamsplitter 680 DCSPXR, Chroma) onto the back aperture of the objective lens (see Figure 3, \#9) where stray light from the NIR in the detection path is blocked by a short pass filter (2P-Emitter E 700 SP, Chroma). In order to perform spectrally resolved measurements, a filter wheel (La Vision Biotec) or a spectrograph (Triax 190, HORIBA Jobin Yvon) can be introduced into the detection pathway (see Figure 3, \#7). The scanning unit consists of an integrated pre-chirp section (Figure 3, \#2) to compensate for laser pulse dispersion and two galvanometric mirror scanners (Figure 3, \#4) to scan the laser foci in one optical x-y-plane (i.e. one depth) of the sample. The multiplexing 
section (Figure 3, \#3) of the TriM Scope can split up the incident laser beam into a variable number of beams of the same average power. This section consists of a set of ten 100\% reflective mirrors and one (adjustable) 50\% mirror. By introducing the $50 \%$ mirror between the set of $100 \%$ mirrors, as shown in figure 3 , the laser beam can be split up into $1,2,4, . ., 64$ beams resulting in an adjustable number of excitation foci in the sample (see Figure 3 , \#6). In addition, the laser power can be adjusted in order to achieve short acquisition times without photodamage. A mechanical focus drive (MFD, Märzhäuser) in combination with a NIR coated objective lens (XLUMPLFL20XW, Olympus) with a large working distance (WD = $2 \mathrm{~mm}$ ) allows for the acquisition of depth resolved fluorescence $\mathrm{x}$-y-scans inside the sample. Data acquisition and control is realized via a software package (Imspector, La Vision Biotec) controlling all necessary experimental parameters such as laser attenuator, number of beams, detection filter settings, scanner settings and the focus drive. 5-dimensional fluorescent data sets (including spectral and temporal data axis) are handled and processed with Imspector, Image J [4] or Imaris 4.0 (Bitplane AG) software packages.

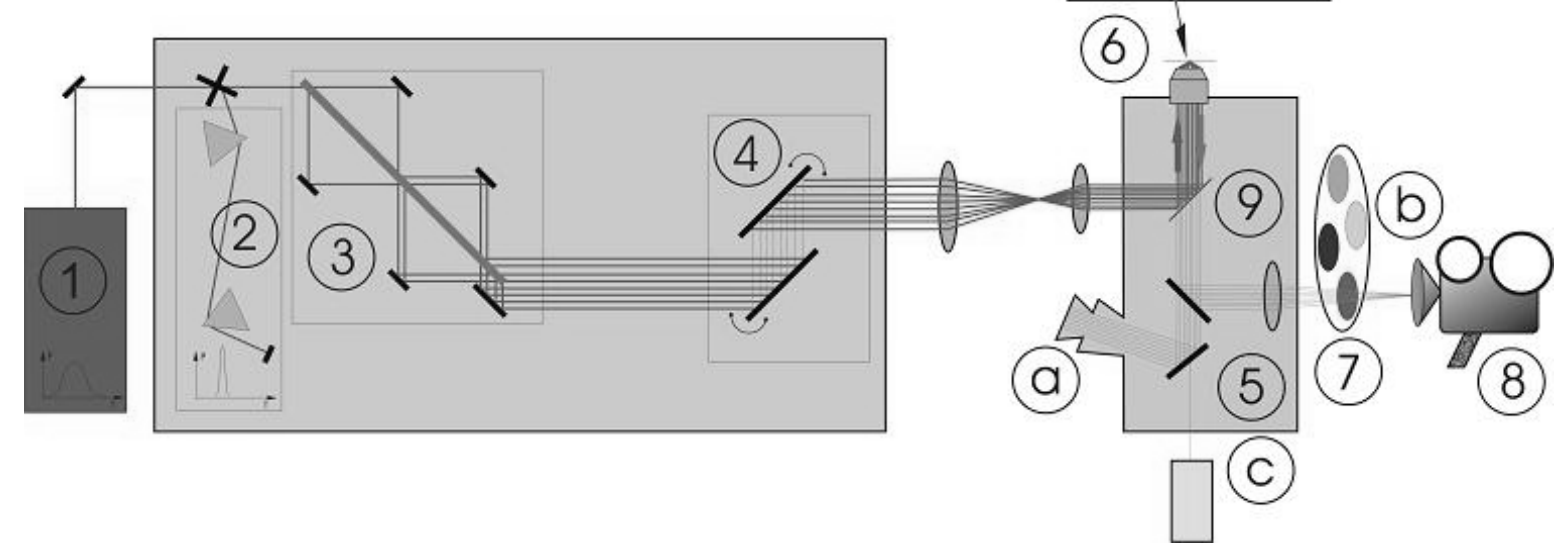

Figure 3: Scheme of two-photon laser scanning microscope

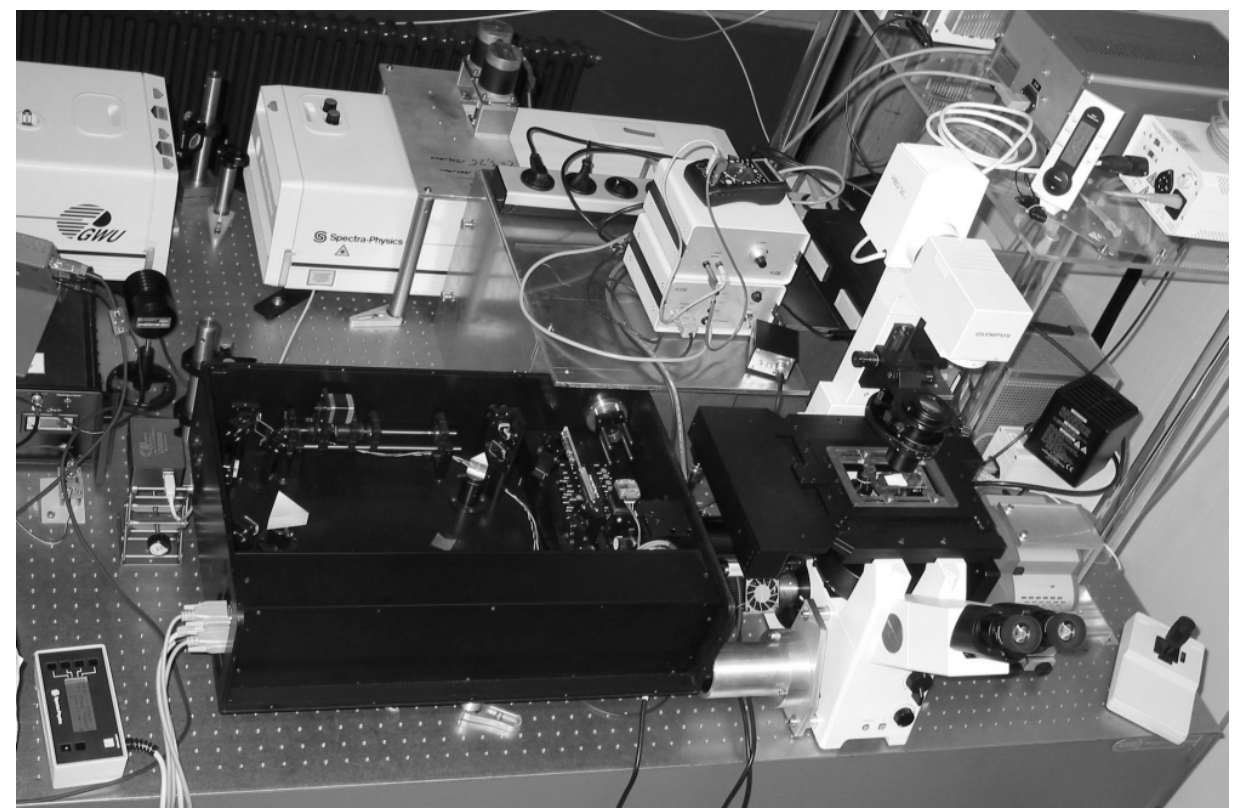

Figure 4: Experimental setup of two-photon laser scanning microscope 


\subsection{Human Cartilage Tissue}

Human cartilage tissue has been received in healthy as well as in arthritic form directly from the surgical ward at a local hospital (Franziskus-Hospital, Bielefeld). Following biopsy, the tissue was kept at room temperature in Ringer solution and investigated within 6 hours after extraction. For sample preparation the tissue was cut with a scalpel and placed directly on a standard microscope glass slide and mounted onto the scanning stage of the inverted 2PLSM. During the transport, preparation and experiment the tissue was always kept in the solution (Ringer). No additional staining or fluorescence labelling was applied.

\section{RESULTS}

Figure 5 shows the 3D-autofluorescence reconstruction of unstained healthy human cartilage as measured with 2PLSM. Measurements with a tissue penetration depth of up to $200 \mu \mathrm{m}$ inside the sample (up to $460 \mu \mathrm{m}$ are possible for arthritic cartilage) at a spatial resolution of approximately 1 micron can be performed within minutes. The image was taken in the 2PLSM mode at an excitation wavelength of $800 \mathrm{~nm}$ with 64 parallel foci at a total laser power of $260 \mathrm{~mW}$, keeping laser power at $4 \mathrm{~mW}$ per focus to prevent photodamage [5]. The spectral discrimination between ECM and chondrocytic cells was achieved by recording two separate microscopy image stacks with fluorescence emission filtering for ECM

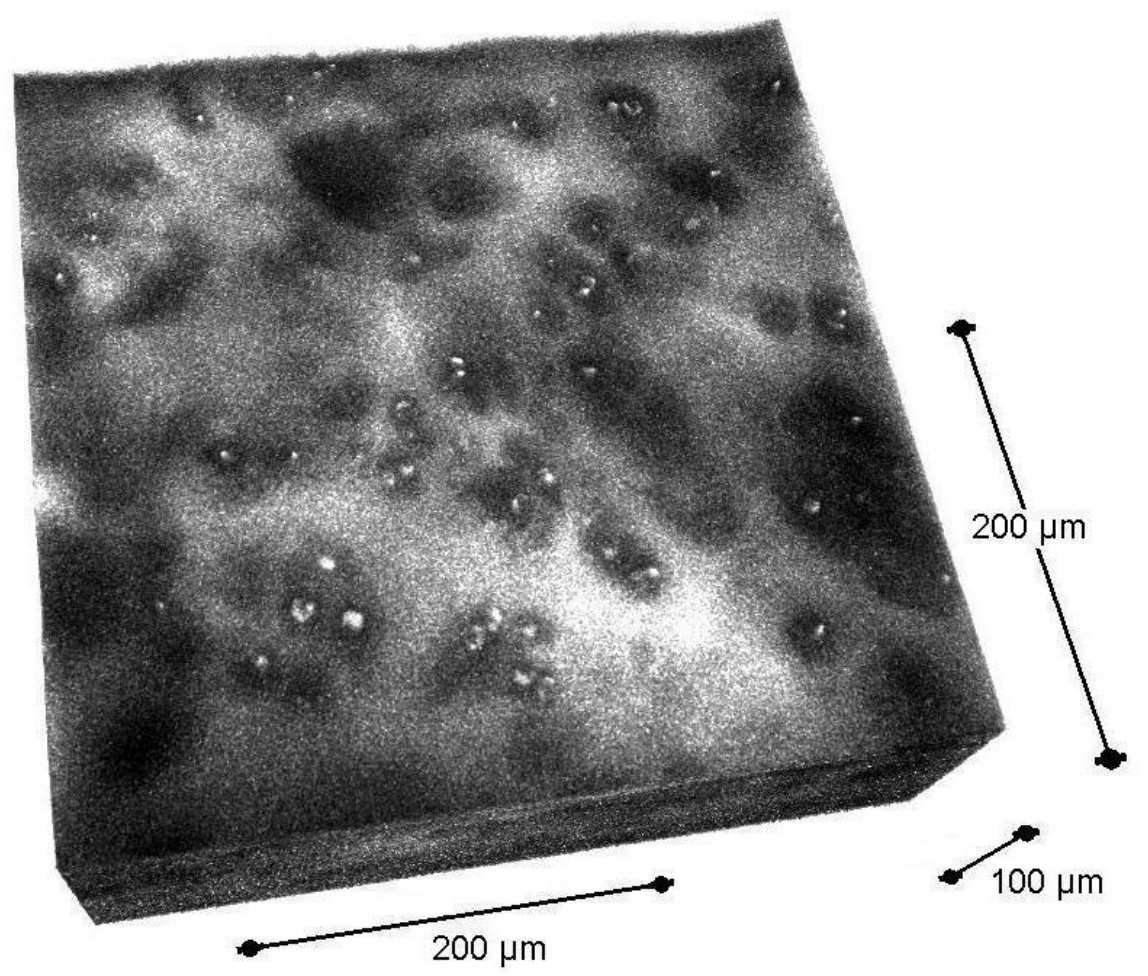

Figure 5: 3D-autofluorescence reconstruction of unstained healthy human cartilage tissue. The ECM and the chondrocytic cells, originally represented in green and red colors, are shown in greyscale representation for printtechnical reasons. The presented plane lies 50 microns below the tissue surface of the sample. 
(HQ 525/50) and for the chondrocytes (HQ 575/50). The superimposed image stack in figure 5, however, was printed in greyscale representation for technical reasons.

From the image in figure 5 it is evident that a spectral discrimination between ECM and chondrocytes is readily possible. Furthermore, the complete mapping of the tissue allows a direct estimate of the corresponding chondrocyte density in the tissue region displayed in figure 5, which was found to be approximately $20 \cdot 10^{6} / \mathrm{cm}^{3}$. This result compares well with values for healthy cartilage found by other research groups [6]. It has to be mentioned thought that the chondrocyte density varies vastly for different samples, even within the same sample, depending on the relative position of the region under investigation in the cartilage. We found chondrocyte densities that range from approximately $2 \cdot 10^{6} / \mathrm{cm}^{3}$ to $20 \cdot 10^{6} / \mathrm{cm}^{3}$ in the same cartilage sample. In addition, the lacunae in the ECM could be identified by a reduced fluorescence signal in colocalization with the grouped chondrocytic cells.

We also investigated healthy and arthritic tissue samples (macroscopical diagnosis) from the same female test person using 2PLSM in order to determine if any differences can be identified (Figure 6). Both tissue samples were characterized with 2PLSM at $800 \mathrm{~nm}$ with a laser power of $240 \mathrm{~mW}$ in 64-foci parallel mode of operation. For mapping the surface morphology here, only the green fluorescent emission filtering for the ECM (HQ 525/50) was recorded.
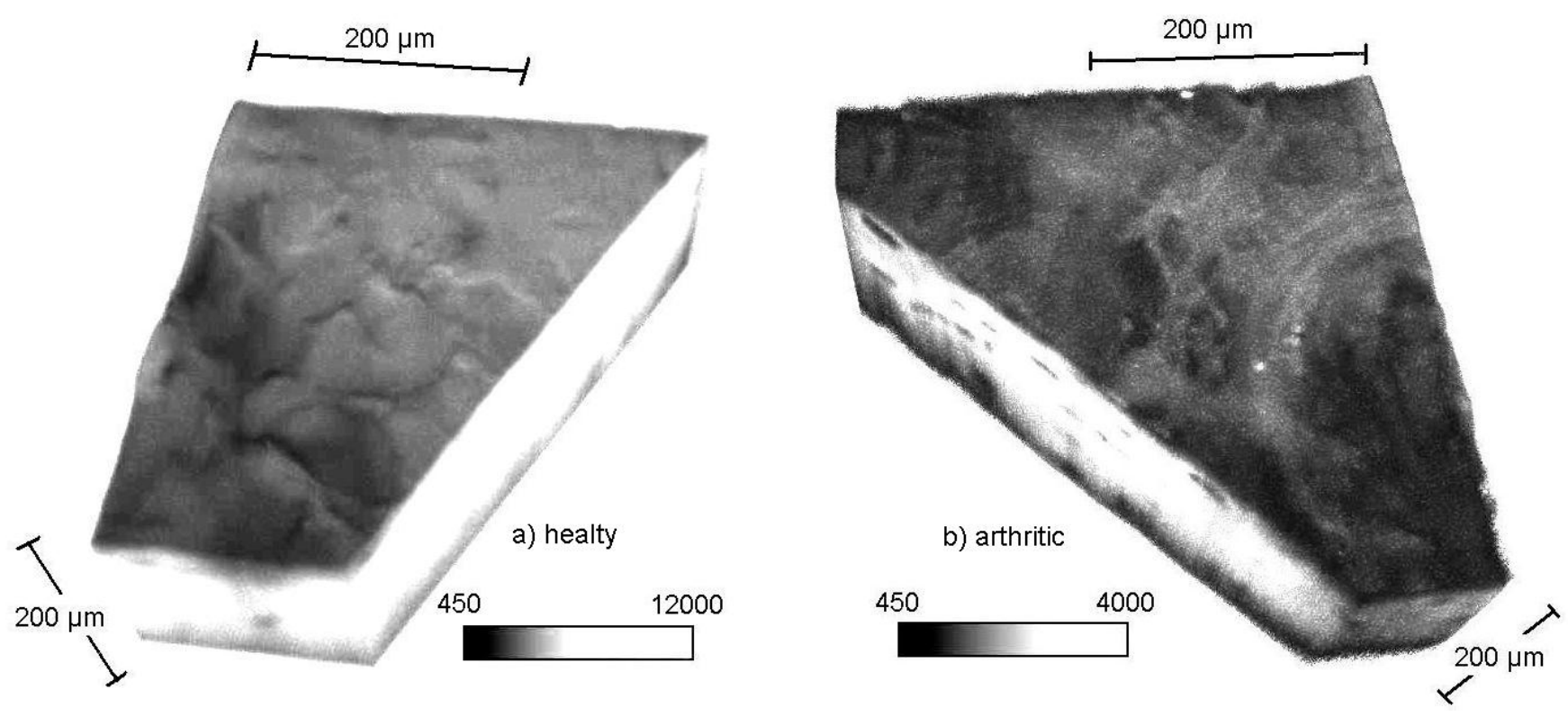

Figure 6: 3D-autofluorescence reconstruction of the surface of unstained healthy and arthritic human cartilage tissue from the same proband. The ECM is shown in grayscale representation.

In figure 6 two things are evident: firstly, healthy cartilage tissue displays a much higher autofluorescence emission from ECM than arthritic tissue, and secondly, the two outer surface structures significantly differ with respect of smoothness and morphology. Whereas the surface of healthy tissue is smooth and isotropic, the arthritic surface is fibrous and rough. This change in morphology of arthritic tissue to a rough, fibrous surface is consistent with an increased friction and resulting wear between cartilage surfaces in contact. Furthermore the reduced fluorescence emission intensity for arthritic tissue can be interpreted as an indication of lower tissue density. 


\section{SUMMARY \& OUTLOOK}

Native hyaline cartilage from a human knee joint was directly investigated with laser scanning microscopy via 2-photon autofluorescence excitation with no additional staining or labelling protocols. Via a spectral autofluorescence separation these experiments allowed discrimination of the chondrocytes from the ECM and therefore an estimate of chondrocytic cell density within the cartilage tissue. Furthermore, a comparison between healthy and arthritic cartilage tissue exhibited distinct differences in tissue morphology and, via relative autofluorescence intensity and penetration depth, distinct differences in ECM density. These findings can potentially be used in future diagnostic applications, for example for a better quantitative definition of different arthritic states of articular cartilage.

\section{Acknowledgements}

We thank V. Andresen, G. Hildebrand, K. Hinterkeuser, V. Kunert, K. Liefheit, and R. Schade for technical support and valuable discussions. Financial support from BMBF in the Biophotonics Research Initiative (Grant MEMO (FKZ: 13N8432)) is gratefully acknowledged.

\section{REFERENCES}

[1] Smith JO, Oreffo RO, Clarke NM, Roach HI. "Changes in the antiangiogenic properties of articular cartilage in osteoarthritis" J Orthop Sci. 2003;8(6):849-57

[2] http://cal.vet.upenn.edu/saortho/chapter_05/05mast.htm

[3] W. Denk, J. H. Strickler, W. W. Webb, "Two-photon laser scanning fluorescence microscopy", Science, 248, 73-76, 1990.

[4] For ImageJ Reference (http://rsb.info.nih.gov/ij/)

[5] K. König, "Multiphoton microscopy in life sciences”, Journal of Microscopy, Vol. 200, Pt 2, November 2000, pp. 83-104

[6] Hunziker EB, Quinn TM, Hauselmann HJ,” Quantitative structural organization of normal adult human articular cartilage", Osteoarthritis Cartilage. 2002 Jul;10(7):564-72

[7] J. Martini et al., in preparation, (2005) 